\title{
Aspects microbiologiques des milieux d'estuaires
}

\author{
par M. Aubert \\ Directeur de Recherches à I'I.N.S.E.R.M. \\ Directeur du C.E.R.B.O.M.
}

\author{
$M^{\text {me }}$ J. Aubert \\ Chargé de Recherches à l'I.N.S.E.R.M. \\ Directeur-adjoint du C.E.R.B.O.M.
}

et M. Gauthier

Chargé de Recherches à I'I.N.S.E.R.M.

C.E.R.B.O.M. - I.N.S.E.R.M. (U. 40), Nice (France)

Après l'exposé de $M$. le Professeur Pérès, point n'est besoin de revenir en détail sur la définition des estuaires et leur écologie générale.

Cette définition, à première vue facile à formuler, demeure aujourd'hui encore très discutée. A la limite, la mer du Nord, dans son ensemble, n'a-t-elle pas été en effet considérée par certains comme un estuaire? Nous n'avons pas l'intention ici d'ajouter à cette discussion. Il nous paraît cependant nécessaire de préciser qu'il convient d'inclure, dans l'ensemble des milieux d'estuaires, toutes les zones d'extension en mer des eaux usées provenant d'usines ou d'agglomérations littorales. On retrouve en effet, dans ces aires, un ensemble de caractéristiques physiques, chimiques et biologiques propres aux estuaires.

Cette précision apportée, nous restreindrons cette étude aux phénomènes purement bactériologiques, compte tenu évidemment des interactions considérables existant entre le monde bactérien et l'ensemble des autres organismes du milieu.

Deux grands groupes de micro-organismes peuvent être distingués :

- tout d'abord les micro-organismes « autochtones», propres au milieu d'estuaire considéré dans un état d'équilibre dynamique normal;

- et les micro-organismes exogènes, ou «polluants», souvent d'origine fécale, apportés par les eaux douces terrigènes.

La microbiologie des estuaires présente donc deux aspects relativement distincts mais intimement liés puisque, du fait de la plus ou moins grande dilution des eaux marines épuratrices, on assiste dans ces milieux à une plus longue survie des bactéries terrestres. Bien qu'assez mal connue dans le détail, son étude peut être abordée d'une manière analytique en considérant successivement :
- les conditions physico-chimiques qui règnent au sein de ces milieux;

- la nature et le métabolisme des espèces microbiennes qui les peuplent;

- les interactions qui existent entre ces espèces et le milieu d'une part, entre les micro-organismes eux-mêmes d'autre part.

\section{Facteurs physico-chimiques des estuaires}

Quelques mots seulement pour évoquer les plus importants d'entre eux. Répétons, tout d'abord, que les milieux d'estuaires sont fondamentalement caractérisés par la variabilité des conditions physico-chimiques qui s'y développent. Cette variabilité est cependant beaucoup plus grande dans l'eau qu'au niveau benthique et dans les sédiments.

Parmi les facteurs physiques les plus importants, il faut citer en premier lieu la température, puisqu'elle a une incidence considérable sur le développement des bactéries. Elle est évidemment très variable, beaucoup plus qu'en milieu marin; ses fluctuations sont à la fois locales, journalières et saisonnières, et peuvent s'étendre sur plus d'une dizaine de degrés en l'espace de 24 heures. Leur amplitude est très nettement atténuée en milieu sédimentaire. Si bien que la plupart des bactéries que l'on rencontre dans ces milieux sont eurythermes.

pH et rH sont également très importants et relativement variables. Ils sont, en fait, beaucoup plus contrôlés par les micro-organismes qu'ils ne les contrôlent eux-mêmes. Dans l'eau, le $\mathrm{pH}$ est directement sous la dépendance du jeu entre la respiration des organismes vivants et la photosynthèse. Dans les sédiments la réduction des sulfates 


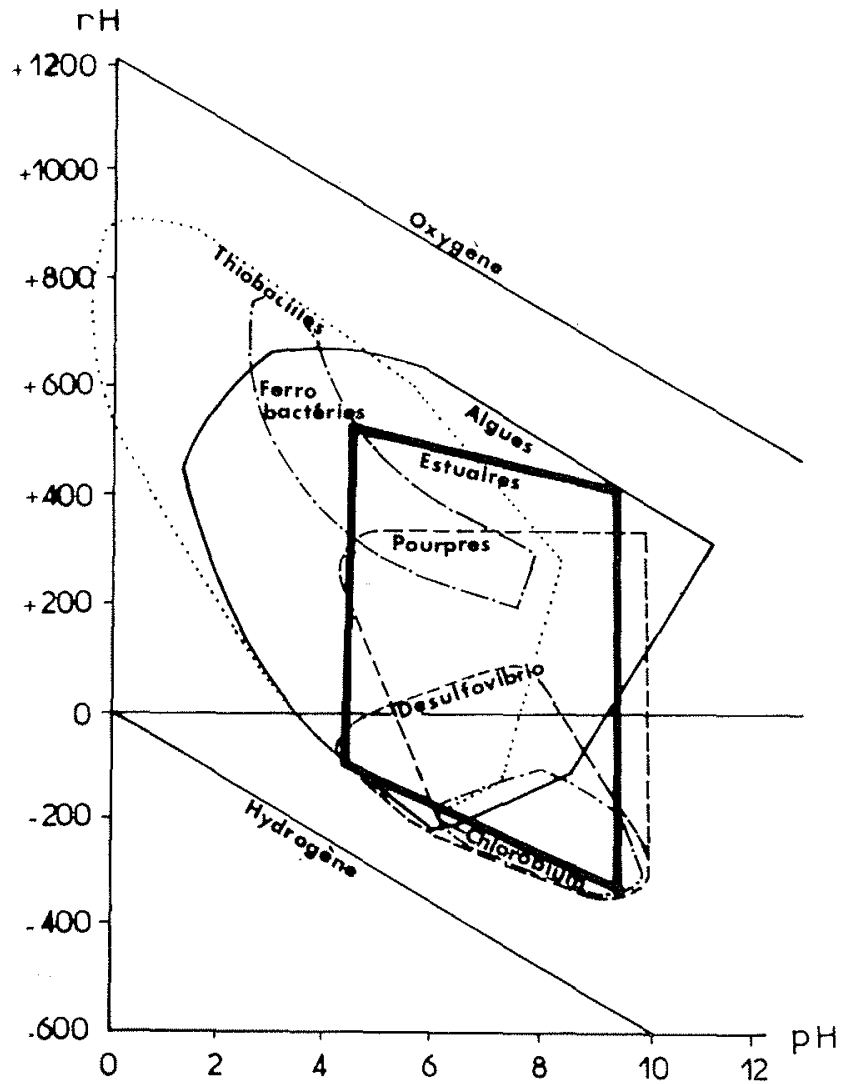

1/ Relations $\mathrm{pH} / \mathrm{rH}$ dans les milieux d'estuaires (d'après E. J. F. Wood, 1967 [3])

en sulfures tend à maintenir le pH globalement aux environs de la neutralité, bien que des fermentations bactériennes puissent localement le rendre plus acide, jusqu'à 6,4 ou moins selon le cas. Le $\mathrm{rH}$ est généralement positif $(+550 \mathrm{mV})$ dans les eaux, et négatif dans les sédiments (jusqu'à $-300 \mathrm{mV}$ ) où la réduction favorise le développement d'une microflore anaérobie très dense. En fait, si l'on considère les relations entre $\mathrm{pH}$ et $\mathrm{rH}$ dans ces milieux d'estuaires, on observe la répartition écologique représentée dans la figure 1 .

Le milieu d'estuaire n'occupe qu'une faible surface par rapport au potentiel biologique des principales espèces bactériennes et algales qui le peuplent. Ceci est dû à l'effet tampon du système carbonate-bicarbonate de l'eau de mer, et aux échanges d'oxygène à l'interface eau-air sous lequel cet oxygène tend à la saturation, ou même à la sursaturation, si ledit milieu est turbulent (Wood, 1967). Le rH de l'eau est, dans certains cas relativement rares, contrôlé par l'activité des bactéries du soufre. Dans les autres cas, il est sous la dépendance de la photosynthèse.

La salinité est un facteur chimique extrêmement important dans l'écologie des estuaires. Ses fluctuations sont considérables, dans le temps comme dans l'espace, puisqu'elles sont liées directement au rapport entre les quantités d'eau douce et d'eau de mer en chaque point, ainsi qu'aux courants, aux climats et à l'évaporation de l'estuaire. M. le Professeur Pérès a, tout à l'heure, longuement montré les gradients qui s'élaborent au sein de ces milieux. Les variations de salinité conduisent évidem- ment à la sélection et à l'établissement de populations bactériennes euryhalines. Elles gouvernent largement la distribution spatiale de ces micro-organismes. Il est pourtant souvent difficile de définir exactement le rôle de cette salinité en milieu d'estuaire : de nombreuses substances chimiques apportées par les eaux terrigènes (éléments traces, substances de croissance, matières organiques floculées) peuvent fort bien y être des facteurs limitants et jouer un rôle beaucoup plus déterminant que la simple dilution saline.

D'autres facteurs naturels concourent également à la répartition des micro-organismes dans ces biotopes. L'éclairement, par exemple, qui agit tout autant au niveau des algues ou du plancton végétal qu'à celui des bactéries photo-autotrophes. Notons que cet éclairement est souvent affaibli par la turbidité des eaux d'estuaires chargées en matières à l'état particulaire. Il faut, en outre, signaler qu'en dehors de l'apport normal de divers éléments minéraux (phosphates, nitrates) ou organiques (acides humiques, substances de croissance), les eaux terrigènes amènent aux estuaires ces polluants chimiques dont on a parlé précédemment : détergents, pesticides, hydrocarbures, métaux lourds et matières organiques qui altèrent profondément l'équilibre biologique du milieu. A l'échelon microbien, leur influence n'est cependant pas obligatoirement défavorable et nous en décrirons quelques effets à la fin de cet exposé.

Leur activité destructrice sur la flore et la faune est bien connue : elle a été évoquée tout à l'heure, et nous en étudierons plus loin quelques aspects plus particulièrement bactériologiques.

\section{Activité bactérienne en milieu d'estuaire}

Pour reprendre la distinction faite au début de cet exposé, nous considérerons d'une part l'activité des bactéries dites «autochtones», propres au milieu naturel, et d'autre part celle des germes «polluants» terrigènes.

\section{Activité bactérienne endogène}

Plus encore qu'en milieu marin, peut-être, il est difficile d'établir l'appartenance spécifique d'une bactérie aux milieux saumâtres d'estuaire. Autant que faire se peut, les microbiologistes s'intéressant au milieu marin ont tenté de déterminer les besoins des bactéries pour les ions majeurs de l'eau de mer et, sur la base de ce critère, de les différencier physiologiqument. Ainsi, ils considèrent que l'on peut diviser l'ensemble des bactéries en trois grands groupes selon leurs besoins en sodium :

— «marines» sont celles qui nécessitent d'une manière absolue la présence de sodium à une concentration supérieure à $0,2 \mathrm{M}$;

— «semi marines» celles qui se développent en présence de concentrations en $\mathrm{Na}+$ comprises entre 0,005 et $0,2 \mathrm{M}$;

- et «terrestres» celles qui peuvent se développer dans des milieux dont la concentration en $\mathrm{Na}+$ est inférieure à $0,005 \mathrm{M}$. 
Les bactéries des milieux saumâtres appartiennent toutes aux deux derniers groupes. En fait, dans ce milieu, et à quelques exceptions près, température et salinité permettent en principe une survie relativement longue de très nombreuses bactéries terrestres aérobies ou anaérobies. Certaines d'entre elles, eurythermes et euryhalines, sont tout à fait capables de s'y intégrer et de participer activement aux cycles de la matière. Les seules bactéries terrigènes réellement "polluantes», au sens écologique et épidémiologique du terme, sont les bactéries parasites des organismes vivants terrestres et, en particulier, les espèces pathogènes : leurs exigences nutritionnelles sont telles qu'elles sont incapables de s'insérer dans les cycles vitaux des eaux saumâtres. Leur destinée sera évoquée plus loin.

Dans l'eau des estuaires, les germes aérobies abondent. Ils appartiennent aux genres les plus représentatifs des microflores naturelles : Pseudomonas, Vibrio, Aeromonas, Achromobacter, Agrobacterium, Flavobacterium, Erwinia, Xanthomonas, Chromobacterium, Cytophaga parmi les gram négatifs; Bacillus, Micrococcus, Staphylococcus, Streptococcus, Sarcina, Corynebacterium parmi les gram positifs les plus fréquents. Ils sont naturellement liés à de nombreux micro-organismes plus évolués : Actinomycètes, levures, champignons inférieurs, algues unicellulaires et cyanophycées. Ces bactéries aérobies se développent selon les voies classiques des cycles de la matière en aérobiose : dégradation des matières organiques avec oxydation progressive du carbone jusqu'au stade du $\mathrm{CO}_{2}$ le plus stable, fixation d'azote, nitrification ou dénitrification, etc.

Ce milieu aqueux peut, dans certaines conditions dystrophiques ou de stagnation, devenir totalement anoxique. Dans ce cas, s'y développent de nombreuses espèces anaérobies hétérotrophes : Clostridium, Plectridium, Bacillus, Vibrio, Veillonella, etc.; photo-autotrophes : bactéries sulfureuses vertes (Chlorobium, Chloropseudomonas), bactéries sulfureuses pourpres (Chromatium et seize autres genres) et bactéries pourpres non sulfureuses (Rhodomicrobitum, Rhodosporillum); ou chimio-autotrophes : nitrifiantes (Nitrisomonas, Nitrobacter), sulfo-oxydantes (Thiobacillus) et hydro-oxydantes (Hydrogenomonas).

Plus particulière, et moins bien connue, est la vie anaérobie qui se développe dans les sédiments. La flore bactérienne, très complexe, comporte l'ensemble des espèces anaérobies citées ci-dessus, ainsi que de très nombreuses autres espèces auto ou hétérotrophes participant à certains cycles endogènes particuliers (ferrobactéries, méthanobactéries, etc.).

Dans les sédiments, ce sont ces bactéries qui règlent le $\mathrm{pH}$ et le $\mathrm{rH}$ : de ce fait, elles jouent un rôle fondamental đans l'écologie de ces milieux. La limite alcaline du $\mathrm{pH}$ est fixée par le $\mathrm{CO}_{2}$ et l'équilibre carbonates-bicarbonates, lui-même réglé par l'équilibre des ions $\mathrm{CA}++$ et $\mathrm{Mg}^{++}$; l'acidité la plus basse possible est contrôlée par la production d'ion $\mathrm{SO}_{4}^{--}$due aux bactéries sulfo-oxydantes (G. Thiobacillus). Le potentiel d'oxydo-réduction est généralement gouverné par les micro-organismes photosynthétiques, ou bien encore, dans certaines aires, réduites par les ferrobactéries. La réduction des sulfates (Desulfovibrio) et l'activité de certains autres anaérobies hétérotrophes peuvent diminuer le $\mathrm{rH}$ jusqu'à $-300 \mathrm{mV}$ par formation d'ion sulfure $\mathrm{SH}^{-}$. Cet ion peut être réoxydé anaérobiquement, en l'absence d'oxygène moléculaire et à la lumière, par les bactéries sulfureuses vertes en $S^{--}$.
Quand les ion $\mathrm{OH}^{-}$sont présents dans le milieu, les bactéries sulfureuses pourpres et Thiobacillus denitrificans apparaissent et oxydent le soufre et les sulfures en sulfates.

Par ailleurs, la chimie des détritus organiques, et ses relations avec les micro-organismes hétérotrophes dans les sédiments ou dans l'eau sont complexes, peut-être encore plus que pour les organismes autotrophes. On cherche encore, à l'heure actuelle, des méthodes de mesure de la décomposition des matières organiques, en particulier pour les sédiments. Une méthode micro calorimétrique, par exemple, pourrait apporter de précieux renseignements sur la physiologie microbienne endogène de ces milieux. Il est bien connu que, en anaérobiose, les composés carbonés peuvent, au stade ultime, être réduits en méthane et hydrogène par les méthano-bactéries (exception faite pour la méthionine, qui est réduite en diméthylsulfure). Le dégagement de méthane ou d'hydrogène à l'état libre est cependant chose rare : généralement, ces deux molécules sont réoxydées dans l'environnement immédiat de leur zone de formation. Par contre, à la surface du sédiment ou dans l'eau, la dégradation des composés organiques est réalisée le plus fréquemment jusqu'au stade d'oxydation le plus stable $\left(\mathrm{CO}_{2}\right)$, ou bien s'arrête à celui de certains composés intermédiaires (lactate, acétate, pyruvate). Les composés azotés peuvent être réduits en $\mathrm{NH}_{3}$; en fait, eau et sédiment sont souvent très riches en nitrates, mais l'azote est en majeure partie utilisé sous forme d'ammoniaque.

Il serait vain, et en fait inutile, de vouloir rentrer ici dans le détail des activités bactériennes qui régissent l'ensemble des cycles de la matière dans l'eau ou les sédiments d'estuaires. Une telle étude nous conduirait trop loin. Nous reviendrons cependant plus loin sur certains d'entre eux (en particulier celui des bactéries méthanogènes) qui sont impliqués dans les processus de transfert de certains polluants chimiques métalliques dans les chaînes alimentaires.

Voyons maintenant quels sont les aspects et les conséquences de la pollution bactérienne et chimique dans les milieux d'estuaires.

\section{Pollution microbienne}

\section{a) Nature des éléments polluants}

Les eaux usées des villes sont extrêmement chargées en micro-organismes ( 1 à $10.10^{6}$ bactéries par exemple par millilitre en moyenne), et cette microflore comprend un bon nombre d'espèces pathogènes (virus, leptospires, champignons, levures, bactéries, protozoaires et certains œufs de métazoaires).

Ces micro-organismes sont présents en quantités extrêmement variables d'un estuaire à l'autre, mais on les retrouve d'une manière constante dans les estuaires fortement pollués, ce qui n'est pas sans importance pour la santé publique dans ces régions.

Il existe une énorme masse de travaux, effectués surtout en Europe et aux U.S.A., traitant à la fois du problème de la survie de ces micro-organismes pathogènes en milieu marin ou saumâtre, et de celui des facteurs d'épuration dans ces milieux. Si peu de choses sont encore connues en ce qui concerne la survie des œufs ou des kystes de micro-organismes pathogènes, on connaît par contre assez 
bien les temps de survie des bactéries et des virus en eau de mer plus ou moins diluée. Généralement, les bactéries à gram positif sont nettement plus sensibles et disparaissent plus rapidement. Les autres survivent pendant des temps variables selon l'espèce, le taux d'eau de mer et la nature ainsi que la quantité d'eau usée : généralement de l'ordre d'une à deux semaines, mais souvent en moins de trois jours, pour Salmonella, Escherichia coli, les coliformes. Dans la plupart des cas, 90 à $99 \%$ des bactéries sont éliminées dans les deux à trois premiers jours, le reliquat persistant parfois beaucoup plus longtemps. Certaines espèces peuvent, en outre, s'adapter plus facilement au milieu et y persister durant des périodes plus longues, surtout dans le cas des estuaires. C"est, en particulier, le cas des clostridies des gangrènes, de Vibrio parahaemolyticus et de Clostridium botulium type E. Quant aux virus, ils sont généralement inactivés, mais après des temps de contact plus longs.

\section{b) Auto-épuration}

La réalité du phénomène d'épuration en estuaire a été établie sans équivoque par Ketchum (1952) au cours d'une étude comparative de la diminution théorique (dilution pure) et réelle (numérations directes) du nombre des bactéries coliformes dans l'estuaire de la Raritan River. Sur la figure 2, la différence de pente, entre les deux courbes, établit d'une manière indiscutable la réalité de l'épuration biologique du milieu. Ce résultat est d'ailleurs tout à fait comparable à ceux obtenus par M. Aubert et J. G. Bonde en mer ouverte, respectivement en Méditerranée et en mer Baltique.

Quelles sont les facteurs qui sont responsables de cette auto-épuration?

L'ensemble des données connues à l'heure actuelle dans ce domaine a été structuré par M. Aubert dans une Théorie générale de l'auto-épuration de la mer. Selon ces données, on doit considérer ici deux ensembles de facteurs concourant à l'épuration des milieux d'estuaires, dont l'origine est cependant profondément différente :

- des éléments épuratifs présents dans les eaux usées elles-mêmes, donc d'origine terrestre;

- des facteurs d'épuration propres à l'estuaire considéré comme un milieu individualisé, donc endogènes.

Dans les eaux usées et l'ensemble des eaux douces naturelles plus ou moins polluées, existent des micro-organismes «prédateurs » qui se développent au détriment des populations bactériennes : ce sont les virus bactériophages et certains micro-prédateurs, nouvellement découverts par Stolp et coll., puis étudiés par A. Guélin et M. Shilo, qui portent le nom de Bdellovibrio bacteriovorus. Ces petits organismes (1/10 de micron de longueur), beaucoup plus proches des bactéries que des virus, détruisent les bactéries par choc mécanique et se nourrissent à partir des substances cytoplasmiques. Ils sont extrêmement répandus dans ces eaux polluées et y jouent, très certainement, un grand rôle bactériolytique. Leur activité semble cependant devoir être minimisée à long terme car :

- comme les virus, ils sont inactivés lentement par l'eau de mer (Daniel, 1967);

- ils sont beaucoup moins actifs pour des concentrations bactériennes faibles (Guélin, 1972).
En outre, dans les eaux usées, un grand nombre de Protozoaires se nourrit par ingestion de bactéries et concourt ainsi à l'épuration bactérienne. Cette absorption ne se fait d'ailleurs pas au hasard, certaines espèces bactériennes étant «préférées» à d'autres, apparemment plus toxiques (Chang et coll.).

Les facteurs épuratifs propres au milieu d'estuaire naturel sont beaucoup plus variés :

- Facteurs physiques : communs à tous les milieux aqueux naturels; c'est le cas de la lumière solaire globale qui inactive les bactéries ou qui permet le développement de certains organismes photosynthétiques antibiotico-producteurs. C'est aussi le cas du rayonnement ultra-violet solaire, qui n'agit cependant que dans la couche d'eau d'extrême surface;

- Facteurs chimiques : pH et $\mathrm{rH}$ sont souvent défavorables à la survie des pathogènes dans les estuaires. La température y est également trop faible. Ces facteurs ne sont pourtant pas responsables de la mort des bactéries, tout au plus rendent-ils le milieu hostile à ces germes;

- Facteurs biologiques : les organismes vivants jouent dans l'épuration des estuaires un rôle prépondérant. Ils peuvent agir directement, comme l'ensemble des invertébrés filtrants «suspension» ou deposit feeders, qui se nourrissent des bactéries de l'eau, du film à la surface du fond ou de celles du sédiment même. C'est, en particulier, le cas de tous les mollusques dont les capacités de filtration sont considérables (une moule peut filtrer plusieurs litres d'eau par heure).

Ils peuvent également épurer le milieu indirectement, par l'intermédiaire de sécrétions antibactériennes. On retrouve ce type d'activité épuratrice :

- Chez certains organismes benthiques animaux : éponges (Nigrelli, 1959), our mollusques (Prescott, 1962-1965).

- Chez de nombreuses algues supérieures présentes en milieu d'estuaire comme en milieu marin côtier. Sieburth en a effectué une étude particulièrement exhaustive, et signale de tels phénomènes chez de nombreuses algues vertes (Ulva, Enteromorpha, Cladophora, Codium, etc.), ou rouges (Ectocarpus, Padina, Desmarestia, Laminaria). L'activité antibactérienne est due à divers composés chimiques tels que : acide acrylique naissant, dérivés des chlorophylles, tanins, phénols, terpènes, acides gras, parfois même acide sulfurique (Desmarestia).

- Chez certaines algues planctoniques euryhalines et eurythermes, que l'on retrouve couramment en milieu d'estuaire dans les zones à salinité relativement importante (Asterionella japonica, Skeletonema costatum), ou dans celles à salinité beaucoup plus faible (Chlorella, Scenedesmus). Une importance particulière est à accorder à l'espèce Asterionella japonica, qui peut apparaître en quantités énormes (plusieurs centaines de millions de cellules par millilitre d'eau) et qui produit deux substances antibactériennes particulièrement bien étudiées au C.E.R.B.O.M. : un nucléoside (voisin de la puromycine) et un acide gras en $\mathrm{C} 20$ photo-activable.

- Chez les bactéries endogènes, dans l'eau, à la surface des substrats immergés ou dans les sédiments. Nombreuses sont, en effet, les souches bactériennes qui pro- 
duisent des substances antibiotiques, souvent de nature macromoléculaire (Gauthier, 1969-1970) et plusieurs dizaines d'entre elles ont été isolées dans des sédiments d'estuaires (Tourneur, 1969). Ces substances sont, en fait, vraisemblablement impliquées dans l'ensemble des interactions entre les bactéries au sein des milieux naturels où elles représentent, probablement, l'une des causes qui régissent l'établissement et l'équilibre des populations microbiennes.

Cet ensemble de facteurs physiques, chimiques ou biologiques devrait permettre une épuration bactérienne à peu près totale des estuaires. Elle ne peut cependant s'effectuer que lorsque les populations naturelles sont préservées dans leur intégrité. Un simple coup d'œil aux estuaires des côtes françaises permet de se convaincre que cet état n'existe plus. Là comme ailleurs, l'équilibre dynamique a été rompu, les populations indigènes profondément remaniées par les pollutions chimiques, et les capacités d'épuration biologiques à peu près annulées.

Voyons rapidement quelles ont été les conséquences des pollutions chimiques au niveau des populations bactériennes.

\section{Conséquences microbiologiques des pollutions chimiques}

D'un point de vue très global, les pollutions chimiques peuvent avoir deux types de conséquences sur la microflore des estuaires :

- une action de sélection des espèces, ramenées à un nombre très faible par l'action toxique directe de chacun des polluants chimiques, ou de la synergie de quelques-uns d'entre eux;

- une eutrophisation massive des milieux, pouvant être suivie d'une dystrophie rapide, avec consommation totale de l'oxygène et l'envahissement par certaines bactéries anaérobies (bactéries sulfato-réductrices) qui intoxiquent ces milieux irréversiblement : c'est malheureusement l'histoire de nombreux estuaires, où l'apport toujours accru de matières organiques ou minérales (nitrates et phosphates) a livré le milieu à l'anaérobiose, apanage exclusif du monde bactérien.

Les conséquences des pollutions chimiques sur l'évolution des populations bactériennes des estuaires sont très importantes, ainsi d'ailleurs que le rôle de celles-ci dans le devenir de divers éléments polluants.

Dans ce domaine, encore plus que dans celui des pollutions bactériennes, les travaux sont innombrables et les résultats très divers. Il serait vain de vouloir faire rapidement une étude exhaustive de nos connaissances à ce sujet. Nous nous contenterons d'exposer un certain nombre d'exemples ayant trait à l'importance fondamentale de populations bactériennes dans les phénomènes de pollution chimique en milieu d'estuaire. Cette importance tient au fait que, en milieu sédimentaire comme dans l'eau, les bactéries représentent toujours l'échelon primordial des cycles vitaux et des chaînes alimentaires. C'est grâce à elles que de nombreux cycles de la matière sont possibles. Il en est de même pour ceux de nombreux éléments chimiques polluants, en particulier les métaux lourds, qui subissent au niveau des estuaires des modifications d'une importance fondamentale.

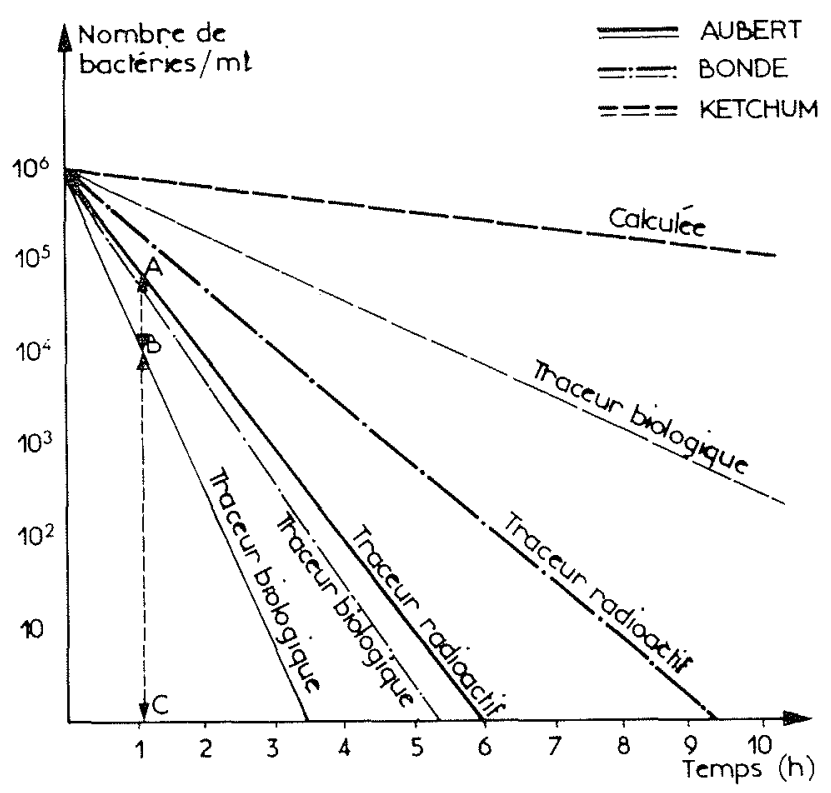

2/ Courbes de diminution théorique et réelle du nombre des bactéries à partir d'un point de rejet pollué, en milieu d'estuaire (B. H. Ketchum, Raritan River, U.S.A.) ou en milieu marin (N. Aubert, Méditerranée; J. Bonde, mer Baltique).

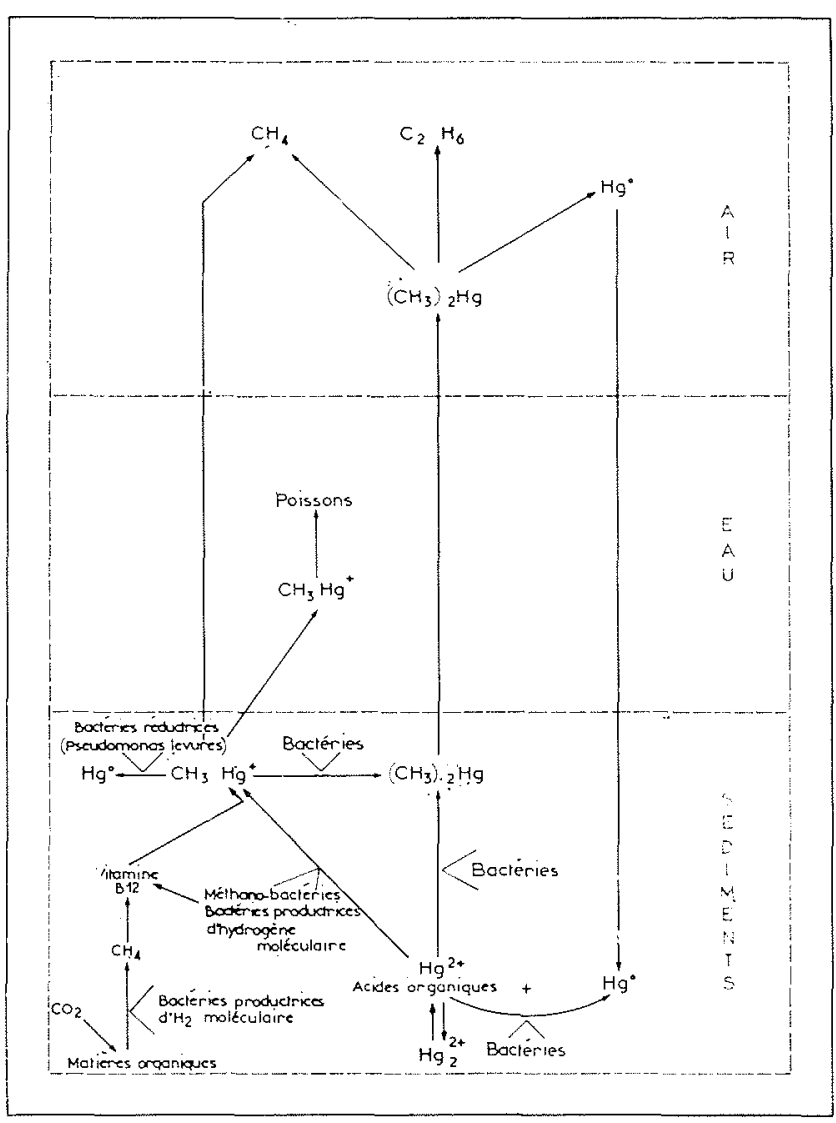

3/ Cycle du mercure (d'après J. Wood). 
Le cas du mercure est particulièrement caractéristique à cet égard (fig. 3). Dans les sédiments des estuaires qui reçoivent certains effluents industriels, le mercure se trouve sous forme $\mathrm{Hg}_{2}{ }^{2}+$ insoluble, en équilibre avec $\mathrm{Hg}^{+}+$qui peut réagir avec les acides organiques (acétique, propionique, butyrique) et les produits de fermentation des bactéries anaérobies. Ces sels mercuriques organiques, très solubles, sont directement utilisables par les microorganismes. Certains d'entre eux prélèvent les ions $\mathrm{Hg}^{++}$ et les convertissent en diméthyl-mercure volatil qui quitte le sédiment et passe dans l'eau, puis dans l'atmosphère. Sous l'action des rayons ultra-violets, le diméthyl-mercure y est converti en méthane, éthane et mercure métal, qui retourne éventuellement dans les sédiments. D'autres bactéries (Methanobacillus, Clostridium) convertissent l'ion $\mathrm{Hg}^{+}+$en méthyl-mercure très toxique qui s'accumule dans les chaînes alimentaires. La transformation de l'ion mercurique en méthyl-mercure se fait par l'intermédiaire de processus métaboliques, mettant obligatoirement en jeu la vitamine B 12 (à partir de $10 \mathrm{~kg}$ de sédiment actif on a pu isoler $85 \mathrm{mg}$ de B 12). Les bactéries méthanogéniques permettent le transfert du groupement méthyl de la vitamine B 12 sur le mercure. Cette transformation intervient uniquement dans la couche superficielle des sédiments (ne dépassant pas $3 \mathrm{~cm}$ ).

C'est donc sous sa forme $\mathrm{Hg}++$ que le mercure est assimilable par les micro-organismes et qu'il peut être «organisé » en méthyl-mercure extrêmement toxique. Or, on a pu isoler des bactéries capables de réduire l'ion $\mathrm{Hg}^{+}+$en $\mathrm{Hg}^{\circ}$ (métal), et d'autres (Pseudomonas, levures) qui réduisent le méthyl-mercure en $\mathrm{Hg}^{\circ}$ et méthane. On sait également que la concentration du méthyl-mercure dans les sédiments est liée à la nature des matières organiques et au $\mathrm{pH}$ (optimum à 4,55). Sa libération, à partir du sédiment, dépend beaucoup de la teneur en soufre. Si la teneur en soufre est élevée, le méthyl-mercure a tendance à être retenu, sinon il est relargué dans l'eau sous forme de cation méthyl-mercure et assimilé directement par les onganismes marins où il se fixe dans les muscles, au niveau des groupements sulfhydriles des protéines. C'est sous cette forme organique qu'il est absorbé par l'homme.

Ainsi, la rétention ou la libération du mercure dans les sédiments d'estuaires - et par voie de conséquence sa concentration dans les chaînes alimentaires - dépendent entièrement de l'équilibre entre les populations bactériennes endogènes sédimentaires. Que les conditions physico-chimiques du milieu soient favorables aux espèces réductrices de méthyl-mercure ou de $\mathrm{Hg}^{+}+$et, quelle que soit la concentration en mercure du sédiment, on trouvera peu ou pas de mercure dans la chair des poissons de l'estuaire. Mais il suffit que l'équilibre soit déplacé et que les conditions deviennent plus favorables au développement des méthanobactéries ou de certaines Clostridies, pour qu'apparaisse, en grande quantité, le ređoutable méthyl-mercure qui est immédiatement repris par les échelons supérieurs des chaînes alimentaires. Certains essais ont été tentés pour contrôler artificiellement ces conditions de milieu et maintenir un état favorable aux espèces réductrices de méthyl-mercure; ils ont abouti à un échec. La complexité du problème est encore accrue par la présence dans les estuaires d'autres substances polluantes, qui influent sur les équilibres bactériens et sélectionnent certaines espèces, ou qui agissent parfois en synergie avec le mercure. Ainsi, J. Wood indique qu'il peut y avoir concen- tration du mercure en présence de plomb tétraéthyle, par transméthylation, selon la réaction suivante :

$$
\begin{gathered}
\left(\mathrm{C}_{2} \mathrm{H}_{5}\right)_{4} \mathrm{~Pb}+\left(\mathrm{C}_{2} \mathrm{H}_{5}\right)_{3} \mathrm{~Pb} \mathrm{CH}_{3} \stackrel{\mathrm{Hg}^{2+}}{\longrightarrow} \mathrm{CH}_{3} \mathrm{Hg}^{+} \\
+\left(\mathrm{C}_{2} \mathrm{H}_{5}\right)_{3} \mathrm{~Pb} \leftarrow \mathrm{O} \longrightarrow \mathrm{H} \\
\mathrm{H}
\end{gathered}
$$

Le méthyl-mercure ainsi formé devient alors directement assimilable par les organismes marins. On retrouve ici un aspect tout à fait particulier de la pollution par les hydrocarbures, le plomb tétraéthyle étant formé lors de la combustion des essences enrichies.

Le mélange de nombreuses substances polluantes au niveau des estuaires rend les systèmes extrêmement complexes. De nombreuses interactions s'établissent entre elles, qui aboutissent souvent à l'augmentation de l'absorbalité de certains éléments toxiques. Nous pensons en particulier à la chélation des métaux lourds (par les détergents ou l'EDTA) moins facilement assimilables par les micro-organismes sous forme complexée.

Nous ne pourrions terminer cet exposé sans évoquer, parallèlement, les modifications que pourraient provoquer la présence de certaines substances chimiques polluantes dans les relations interspécifiques qui dépendent de médiateurs chimiques. Rappelons, à ce propos, quelques notions importantes dans ce domaine. Depuis quelques années, d'importants travaux, effectués en grande partie au C.E.R.B.O.M., ont abouti à démontrer l'existence et l'activité médiatrice de substances chimiques organiques qui, produites par certaines espèces, sont capables d'informer chez d'autres espèces diverses modifications métaboliques, à l'image des hormones dans un organisme animal. Ces substances chimiques organiques ont été nommées «télémédiateurs » par M. Aubert, qui met ainsi l'accent sur leur action à distance. Dans ce sens, nous avons montré que la production de nucléoside antibactérien par Asterionella japonica, diatomée fréquente dans les eaux saumâtres, s'arrête quand apparaît dans le milieu, et à des concentrations très faibles, unne protéine émise par un Péridinien, Prorocentrum micans. En fait, ces télémédiateurs peuvent être primaires quand ils agissent directement entre deux organismes (le nucléoside d'Asterionella japonica inhibe la croissance de certaines bactéries terrestres), secondaires (comme la protéine de Prorocentrum micans qui arrête la production de nucléoside chez la diatomée, lui-même responsable de la destruction des bactéries), ou même d'ordre supérieur. Ces phénomènes peuvent également être cycliques et fonctionner en feed back (les bactéries terrestres de l'eau d'égout produisent de la vitamine B 12, qui favorise la croissance des algues, dont certaines formes produisent des antibiotiques inhibiteurs pour ces bactéries).

En considérant l'ensemble de nos connaissances dans ce domaine, on peut estimer que de telles substances régissent en fait un grand nombre de relations trophiques, sexuelles, ou prédatrices, dans les milieux aquatiques. Et il va de soi que ces phénomènes sont tout à fait concevables en milieu d'estuaire, qui représente le terme de passage normal entre le milieu marin et les milieux dulçaquicoles.

Or, certains travaux effectués au C.E.R.B.O.M. ont montré que divers polluants chimiques sont capables de modifier ces signaux, introduisant de ce fait un grave désordre dans l'équilibre entre les espèces vivantes du 
milieu. Ainsi, le fuel domestique bloque l'émission des substances antibactériennes d'Asterionella japonica. Le cadmium semble également diminuer cette synthèse métabolique. Chez les bactéries antibiotico-productrices, les métaux lourds ont divers effets : certains accroissent la production d'inhibiteurs par ces bactéries, d'autres au contraire la diminuent. Peu de travaux ont été réalisés dans ce domaine et bon nombre de ses aspects restent inconnus. Quoi qu'il en soit, on découvre là un aspect aussi fondamental qu'insoupçonné de l'activité délétère des pollutions chimiques, qui déborde naturellement le cadre microbiologique. Le danger de cette activité réside directement dans le fait qu'une telle transformation du milieu aquatique peut être lente et discrète, qu'elle concerne l'ensemble des organismes qui y vivent et, qu'en dernier lieu, elle peut gravement affecter la santé de l'homme.

\section{Discussion}

Président : M. A. BRIN

M. le Président remercie M. Gauthier pour son intéressant exposé et ouvre la discussion.

M. Gras voudrait savoir si le cycle du mercure «dans les trois premiers centimètres de sédiments », décrit par $M$. GauThIER, concerne seulement les estuaires ou s'applique aussi à la mer.

\section{Gauthier donne la réponse suivante :}

C'est là un phénomène tout à fait général qui a été étudié en estuaire et aussi dans certaines zones marines côtières; en l'état actuel de nos connaissances en ce domaine, il semble dépendre entièrement des populations bactériennes.

M. Gauthier cite l'exemple de l'estuaire de la Sacramento River, dans la baie de San Francisco, où l'on retrouve en quantité importante le mercure qui avait été rejeté par les «chercheurs d'or» au siècle dernier. Fort heureusement, on le trouve dans les sédiments à une profondeur assez importante; de ce fait, il n'est pas repris par les chaines alimentaires parce que la vie bactérienne ne joue pas dans ces zones profondes.

M. BANAL intervient en ces termes

$\mathrm{Si}$ j'ai bien compris votre exposé, autrefois lorsque les estuaires étaient vierges de toute souillure, ils constituaient un milieu naturel où l'auto-épuration était particulièrement intense. Mais aujourd'hui, vaut-il mieux rejeter les résidus de la vie urbaine et industrielle dans l'estuaire on directement en mer?

Cela revient, dit M. Gauthier, à poser la question générale et primordiale du rejet des eaux usées, pouvant contenir des bactériespathogènes, sur une côte ou dans un estuaire. Généralement, on estime que le pouvoir auto-épurateur du milieu naturel, qu'il soit estuarien ou marin, est suffisant pour absorber une bonne partie de la pollution bactérienne. C'est-là un phénomène très complexe qui, malheureusement, ne peut plus jouer à l'heure actuelle, dans la plupart des cas, en raison de la dégradation des milieux naturels et de la quantité de bactéries à détruire. On ne peut pas d'ailleurs assimiler le pouvoir auto-épurateur de la mer, pas plus que celui des estuaires, à celui de substances antiseptiques; le processus est totalement différent et nettement plus lent.

L'estuaire n'a pas de propriétés spéciales en ce qui concerne l'auto-épuration, si ce n'est peut-être l'action de ces petits organismes dont je vous ai cité le nom à propos de l'épuration dans l'eau d'égout elle-même. Leur activité bactériolytique est limitée à une zone où cette eau est relativement peu diluée en eau de mer; donc, plus cette zone est longue, plus leur temps d'action est prolongé.

J'ai lu, dit M. Gibrat, à propos de la contamination par les métaux lourds, que la quantité arrivant par lessivage naturel des terrains était nettement plus importante que celle provenant de l'activité industrielle; or, vous ne parlez que de celle-ci. Y-a-t-il eu des études microbiologiques assez anciennes pour éclaircir ce point?

Les données statisitques globales, répond M. Gauthier, ne permettent pas de caractériser les pollutions «ponctuelles» ou locales et de mettre en évidence leur toxicité éventuelle. Or, il est bien évident que, dans un certain estuaire où arrivent deux ou trois rejets d'effluents industriels, la qualité de mercure artificiellement injectée dans le milieu est très supérieure à l'apport provenant du lessivage des terrains.

Je ne pense pas qu'il y ait eu d'étude microbiologique sur des sédiments anciens, permettant de se faire une idée de l'état normal des estuaires avant leur pollution; mais on sait que, cdns certains estuaires actuellement encore préservés de la pollution par le mercure, on n'observe pas de phénomènes pathologiques tels que ceux que j'ai déorits. Les métaux y sont à un niveau de concentration très faible ne constituant pas des polluants; ils agissent comme des «métaux-traces » d'ailleurs nécessaires à la vie. Comme l'arsenic à concentration très faible, les métaux sont des éléments nécessaires, mais à des concentrations beaucoup plus importantes, ils deviennent des polluants parfois même dangereux.

D'après l'exemple du mercure, observe M. LAPICQUE (Laboratoire des Faibles Radio-activités, C.N.R.S.), il semble souhaitable d'établir plusieurs normes de concentration admissibles, selon le type de population bactérienne existant dans le site où est envisagé le rejet. Si l'on s'en tient à une seule norme de concentration limite, il faudrait sans doute adopter la valeur la plus pessimiste.

Le développement des travaux de dragage dans les estuaires, demande M. Fafournoux (Laboratoire Central d'Hydraulique de France), ne risque-t-il pas de remettre en circuit des ions mercure captés dans les sédiments? Dans l'affirmative, il deviendrait nécessaire d'effectuer, avant les dragages, des sondages pour déceler les zones riches en mercure.

Ce sera effectivement, répond $M$. Gauthier, un problème pour les techniciens qui tenteront de récupérer le mercure dans certains estuaires tels celui de la Sacramento River; il faudra absolument éviter que le mercure, contenu dans les sédiments, soit ramené dans les couches supérieures du sédiment ou à la surface, afin qu'il ne soit pas repris par les chaînes alimentaires (et en particulier, par le métabolisme bactérien, phénomène très important dans le cas du mercure).

M. le Président remercie $M$. Gauthier ainsi que toutes les personnes qui sont intervenues dans la discussion et donne la parole à M. BREITTMAYER pour l'exposé de la communication qu'il a établi en liaison avec M. G. LAPICQUE. 